\title{
SIGNIFICADO DE FLUJO DIASTÓLICO AUSENTE O REVERSO EN EL DOPPLER DE ARTERIA UMBILICAL COMO ÚNICA ALTERACIÓN EN LA EVALUACIÓN DE BIENESTAR FETAL EN FETOS CON TRISOMÍA 21
}

\author{
Juan Carlos Manoli M. ${ }^{1}$, Jorge Carvajal C. ${ }^{2}$, PhD. \\ 1 Posgrado de Obstetricia y Ginecología, ${ }^{2}$ Departamento de Obstetricia y Ginecología, Unidad de Medicina Materno \\ Fetal, Facultad de Medicina, Pontificia Universidad Católica de Chile.
}

\section{RESUMEN}

La evaluación del bienestar fetal es un desafío clínico. El hallazgo de flujo diastólico ausente o reverso (FDA/FDR) en la velocimetría Doppler de arteria umbilical es considerado un signo de insuficiencia placentaria. Sin embargo, en fetos con trisomía 21 es posible encontrar FDA/FDR en ausencia de insuficiencia placentaria. Se presenta un caso cínico de una embarazada en cuyo feto se sospecha Síndrome de Down, con velocimetría Doppler de la arteria umbilical con FDA/FDR, sin alteración en otras pruebas de evaluación del bienestar fetal y sin signos de hipoxia al nacer, pero con una cardiopatía congénita. Se han reportado escasos casos similares al expuesto, postulándose que un Doppler umbilical con FDA/FDR puede presentarse como consecuencia de una cardiopatía congénita. Expertos en medicina materno-fetal se han enfrentado a situaciones como la del caso reportado pero no conocen evidencia científica que avale la conducta expectante en estos pacientes. Concluimos que en fetos con Síndrome de Down y FDA/FDR en arteria umbilical debe evaluarse cuidadosamente la presencia de cardiopatías congénitas y mantener la sospecha de insuficiencia placentaria, adoptando decisiones en base a esa sospecha.

\section{PALABRAS CLAVE: Trisomía 21, flujo ausente o reverso en diástole, cardiopatía congénita}

\section{SUMMARY}

Evaluation of fetal well-being is a clinical challenge. The finding of absent or reverse diastolic flow (ADF/ RDF) in the umbilical artery Doppler velocimetry is considered a sign of placental insufficiency. However, it is possible to find $A D F / R D F$ without placental insufficiency in trisomy 21 fetuses. A clinical case of a pregnant woman having a suspected Down syndrome fetus, with ADF/RDF in the umbilical artery Doppler, without any other alteration in fetal well-being tests with no signs of hypoxia at birth, but with a congenital heart disease is reported. Few cases have benne reported showing similar findings, postulating that umbilical artery Doppler with ADF/RDF may be caused by congenital heart disease. Maternal fetal medicine specialist have faced situations like this but they don't known scientific evidence supporting expectant management in these patients. We conclude that fetuses with Down syndrome and ADF/RDF in umbilical artery Doppler should be carefully evaluated by congenital heart disease but keeping a high suspicion of placental insufficiency and acting according to that.

KEY WORDS: Trisomy 21, absent or reverse diastolic flow, congenital heart disease 


\section{INTRODUCCION}

La evaluación de la unidad feto-placentaria (UFP), o del "bienestar fetal", ha sido un desafío desde los comienzos de la obstetricia. A lo largo de la evolución de la especialidad se han desarrollado diferentes métodos y pruebas que pretenden, de la manera más exacta, predecir la presencia o ausencia de bienestar fetal intrauterino. La velocimetría Doppler de la arteria umbilical, en conjunto con otros métodos tradicionales como el registro basal no estresante (RBNE) y el perfil biofísico (PBF), han sido utilizados con éste objetivo para ayudar a definir alternativas de manejo en fetos de riesgo (1). Entre los métodos de evaluación de la unidad feto-placentaria antes descritos, el más importante y que ha permitido disminuir la mortalidad perinatal, es la velocimetría Doppler de la arteria umbilical, específicamente probado usando como medida de resultado el hallazgo de flujo diastólico ausente o reverso (FDA/FDR) (2).

El síndrome de Down es consecuencia de la trisomía del cromosoma 21 (Langdon Down, 1866) y es la única trisomía autosómica de la especie humana de la que sobrevive un número significativo de individuos más allá de un año después del nacimiento. La incidencia global de la trisomía 21 se aproxima a 1 de cada 700 nacimientos; sin embargo, el riesgo varía con la edad materna: $\sim 1$ en 2.000 nacidos vivos en madres de 25 años, 1 en 200 nacidos vivos en madres de 35 años y 1 en 40 nacidos vivos en madres de 40 años. Existen técnicas ecográficas que permiten precisar el riesgo de aneuploidía asignado por la edad materna, como la medición de translucencia nucal y de hueso nasal en la ecografía de 11-14 semanas. Además existen marcadores séricos que pueden sugerir la gestación de un feto con trisomía 21, como alfafetoproteína, estriol no conjugado y fracción libre de subunidad beta de gonadotrofina coriónica humana (3-5).

Se comenta que los fetos con aneuploidías, específicamente trisomía 21 (síndrome de Down), podían presentar alteraciones en el flujo Doppler de la arteria umbilical, con flujo ausente e incluso reverso en diástole, pero en ausencia de compromiso de la unidad feto-placentaria o de la oxigenación fetal, es decir, corresponder a un falso positivo del examen. Reportamos un caso que podría corresponder a esta situación y revisamos si existe evidencia científica que respalde esta aseveración.

Para ese objetivo realizamos una búsqueda en la base de datos PubMed con los siguientes términos Mesh: trisomy fetuses, assessment of fetal well-being y umbilical artery Doppler flow, encon- trándose un reporte de casos clínicos. Como búsqueda secundaria se utilizaron los siguientes términos Mesh: congenital heart diseases, aneuploidy fetuses, umbilical artery Doppler flow. Además se realizó una búsqueda cruzada entre los términos antes mencionados. Se encontraron 136 artículos, de los cuales se seleccionaron 12 por su relación con el objetivo de éste artículo.

Se conversó con tres médicos obstetras, expertos en medicina materno-fetal y con una vasta experiencia en ecocardiografía fetal, quienes dieron su opinión respecto del manejo de este tipo de pacientes. Las preguntas realizadas fueron: ¿se ha enfrentado alguna vez a un feto con trisomía 21 y Doppler umbilical con FDA/FDR sin otra alteración en pruebas de evaluación de bienestar fetal?; ¿cree válido una conducta expectante o conservadora en este tipo de paciente?; ¿conoce literatura que avale esa conducta?

\section{Caso clínico}

Paciente de 40 años con antecedentes de hipertensión crónica sin requerimiento de hipotensores e hipotiroidismo en tratamiento con levotiroxina 125 ug al día, con buen control médico. Multípara de 3 , dos recién nacidos de término y el último de pretérmino con restricción de crecimiento intrauterino severo y flujo ausente en diástole a la velocimetría Doppler de la arteria umbilical que fue interrumpido a las 31 semanas, vía cesárea, por distocia de presentación.

Cursando un embarazo fisiológico con buen control obstétrico; ecografía fetal 11-14 semanas con translucencia nucal (TN) de 1,8 $\mathrm{mm}$, lo que le confería un riesgo de aneuploidía según edad y corregido por translucencia nucal de 1:162. A las 29+3 semanas en control ecográfico rutinario se constató una estimación de peso fetal de $966 \mathrm{~g}$, corresponde a percentil (pc) 10 según curva Juez y pc 10-25 según curva MINSAL, con imagen quística intraabdominal de $15 \times 20 \mathrm{~mm}$ en relación a cámara gástrica, y flujo ausente en diástole al estudio Doppler de la arteria umbilical. Se hospitalizó para estudio, inducción de maduración pulmonar con corticoides y evaluación de la unidad feto-placentaria.

Se realizó ecografía fetal detallada que confirmó imagen de doble burbuja gástrica sugerente de atresia duodenal, hipoplasia de hueso nasal, micrognatia y clinodactilia. Se ofreció cariotipo en muestra de líquido amniótico, pero fue rechazado en decisión conjunta de la paciente y su familia. La ecocardiografía fetal mostró una anatomía sin alteraciones, con función cardíaca conservada y sin signos de hidrops fetal. 
Evolucionó con FDA en arteria umbilical de modo persistente; perfil biofísico diario $8 / 8$, líquido amniótico normal y registro basal no estresante diario reactivo hasta el parto.

Se realizó una biometría a las $31+4$ semanas (15 días después de su ingreso), con estimación de peso fetal de $1.376 \mathrm{~g}$ (pc 2-5 según curva Juez, pc 10-25 según curva MINSAL). Nueva biometría a las 33+4 semanas de gestación constató estimación de peso fetal de $1.526 \mathrm{~g}$ (pc <2 según curva Juez, pc 5 según curva MINSAL).

Se constató trabajo de parto espontáneo avanzado a las 33+4 semanas y se asistió el parto vaginal sin incidentes. Recién nacido de sexo femenino, peso $1.240 \mathrm{~g}$, talla $39 \mathrm{~cm}$, perímetro craneano 28 $\mathrm{cm}$, Apgar 8-9 y sin signos de asfixia fetal perinatal ( $\mathrm{pH}$ en sangre de cordón umbilical 7,21$)$. Al examen físico se constató pliegue simeano en ambas manos, macroglosia e implantación baja de ambos pabellones auriculares. Radiografía de abdomen y evolución clínica compatible con atresia duodenal. Ecocardiografía neonatal mostró comunicación interventricular (CIV), comunicación interauricular (CIA) y ductus arterioso persistente (DAP) con cierre espontáneo, todos sin repercusión hemodinámica. Estudio genético confirmó síndrome de Down.

\section{DISCUSIÓN}

Las alteraciones cromosómicas son responsables de aproximadamente el $10 \%$ de casos de restricción de crecimiento fetal (RCF) (6). En el caso de los fetos con trisomía 21, en general la asociación es con restricciones de crecimiento de carácter leve a moderada (7). La RCF en el contexto de aneuploidía no es secundaria a insuficiencia placentaria.

En el caso presentado hubo una asociación de restricción de crecimiento intrauterino severa y FDA en el estudio Doppler del cordón umbilical. Clásicamente estos hallazgos orientarían a una insuficiencia placentaria como evento fisiopatológico desencadenante $(8,9)$ y se esperaría que el FDA evolucionara a FDR en aproximadamente 7 días. En el caso presentado, el FDA no pareció deberse a insuficiencia placentaria pues permaneció estable por aproximadamente 4 semanas, sin oligohidroamnios (OHA) y sin evidencia de disminución en la oxigenación fetal $(\mathrm{pH} 7,21)$. Es posible que el FDA fuera consecuencia de la cardiopatía congénita, detectada en el período neonatal.

Aneuploidía y FDA/FDR: Un estudio descriptivo en 192 fetos con cariotipo conocido y flujo diastólico ausente en la velocimetría Doppler de arteria umbilical, encontró que el 8,3\% de los fetos tenían una aneuploidía (10). No existen reportes para conocer qué porcentaje de fetos con aneuploidía tienen alguna alteración en el estudio Doppler.

Existe un reporte de 3 casos clínicos (11) de fetos con trisomía 21, restricción de crecimiento intrauterino, alteraciones en el estudio Doppler de arteria umbilical, evaluación de líquido amniótico normal y registro basal no estresante reactivo. El primer caso era un embarazo gemelar dicigoto, secundario a fertilización in vitro. El gemelo $B$ presentó las alteraciones. Se confirmó trisomía 21 pero no se realizó evaluación cardiológica, inicialmente por falta de clínica sugerente y posteriormente por pérdida de seguimiento. En los otros dos casos se confirmó insuficiencia aórtica con ecocardiografía postnatal. En este trabajo se postuló que, si bien una de las principales causas de RCF y alteraciones en el Doppler es la insuficiencia placentaria, las alteraciones estructurales del corazón también podrían producir esas alteraciones en el estudio Doppler, situación que ha sido descrita previamente por otros autores (12). La disminución del flujo sanguíneo anterógrado en la aorta fetal durante el diástole podría explicar por qué hay flujo diastólico ausente o reverso en el estudio Doppler de la arteria umbilical (13).

La asociación entre cardiopatía congénita y alteración del Doppler umbilical ha sido reportada $(14,15)$, pero en general en fetos con cardiopatías congénitas mayores, con alteraciones cromosómicas severas y mal resultado perinatal. En un estudio prospectivo observacional se evaluaron ocho fetos con malformaciones congénitas mayores y cromosomopatías incompatibles con la vida. Todos los fetos fueron seguidos con velocimetría Doppler de la arteria umbilical hasta su muerte intrauterina. En todos los fetos se encontró FDA/FDR, con un intervalo entre ésta alteración y la muerte fetal de 1-7 días (media 3,6 días) (14).

Otro estudio de cohorte evaluó la velocimetría Doppler de la arteria umbilical y cerebral media en 115 fetos con cardiopatía congénita previamente diagnosticada, divididos en 3 grupos. Grupo A, fetos con cardiopatía congénita aislada; Grupo B, fetos con cardiopatía congénita complicados con malformaciones extracardíacas, cromosómicas o no cromosómicas, disfunción uteroplacentaria o hidrops fetal no inmune no cardiogénico; Grupo C, control. Este estudio mostró que en fetos con cardiopatías congénitas muy severas o con malformaciones congénitas de origen cromosómico, el flujo ausente o reverso en diástole en la arteria umbilical puede ser un predictor de mal resultado perinatal, con muerte fetal intrauterina o postnatal (15).

La información reportada permite sospechar que en fetos con trisomía 21 y especialmente si 
existe cardiopatía congénita, es posible encontrar FDA/FDR de arteria umbilical, que no sea causada por insuficiencia placentaria. Sin embargo esta información no permite una conducta expectante o conservadora en fetos con trisomía 21 y velocimetría Doppler de arteria umbilical con FDA/FDR como única alteración en las pruebas de evaluación de bienestar fetal, pues no es descartable la posibilidad de insuficiencia placentaria y así riesgo de hipoxemia fetal.

En la búsqueda de más información se realizó una entrevista a tres expertos médicos obstetras con una vasta experiencia en ecografía y ecocardiografía fetal para conocer como ellos abordan esta situación.

Dr. Crisitian Belmar Jones (16): Refiere que se ha enfrentado a este tipo de pacientes con anterioridad en los cuales no existe alteración alguna en los test de evaluación de la unidad feto-placentaria a excepción del flujo ausente o reverso en el Doppler de arteria umbilical y posteriormente el resultado perinatal es favorable. A su parecer el manejo de estas pacientes debe ser con un seguimiento estricto de la unidad feto-placentaria, con velocimetría Doppler de la arteria umbilical, perfil biofísico y registro basal no estresante tal como se maneja una paciente con sospecha de insuficiencia placentaria, pues no existe evidencia (o por lo menos él no conoce) que sustente otra conducta.

Dr. Luis Medina (17): Refiere haberse enfrentado a esta situación con anterioridad. Su opinión es que es posible manejar de forma más conservadora este tipo de pacientes, solo si existe confirmación genética antenatal de trisomía 21 . Si no tiene confirmación genética previa, un feto con flujo Doppler de arteria umbilical ausente o reverso como única anormalidad en la evaluación de la unidad fetoplacentaria debe ser manejado como una probable insuficiencia placentaria y ser interpretado como un signo de sufrimiento fetal, ya que la disminución en el volumen de líquido amniótico o un registro basal no estresante no reactivo son alteraciones tardías en la evaluación de bienestar fetal. Por último refiere que no tiene conocimiento de literatura que avale la conducta expectante en embarazadas con fetos con trisomía 21 y Doppler umbilical con flujo ausente o reverso en diástole.

Dr. Hernán Muñoz (18): Refiere que se ha enfrentado tanto a este tipo de pacientes como a fetos con FDA/FDR en Doppler de arteria umbilical sin otra alteración en las pruebas de evaluación de bienestar fetal y sin marcadores de aneuploidía. Si bien es cierto que en fetos con aneuploidía los hallazgos al Doppler descritos pueden deberse a cardiopatías congénitas y no a insuficiencia placentaria. Agre- ga que si hablamos de trisomía 21 y no de otras aneuploidías, en general estos hallazgos al Doppler serían secundarios a una disfunción vascular placentaria y por lo tanto no se debería adoptar una conducta expectante o conservadora. Por último señala que, pese a que las conductas adoptadas en el hemisferio norte difieren de las adoptadas en el hemisferio sur respecto al manejo de trisomía 21, no conoce literatura que avale una conducta expectante.

\section{CONCLUSIONES}

Reportamos un caso de una embarazada cuyo feto presenta aneuploidía (síndrome de Down) y velocimetría Doppler de la arteria umbilical con FDA/ FDR, sin alteración en otras pruebas de evaluación del bienestar fetal. El resultado perinatal sugiere que la RCF y la alteración del Doppler no fueron consecuencia de insuficiencia placentaria.

Los fetos con trisomía 21 pueden presentar alteraciones en el estudio Doppler de la arteria umbilical, ya sea con flujo ausente o reverso como única alteración en los test de evaluación de la unidad feto-placentaria, lo que pudiera deberse a una cardiopatía congénita que frecuentemente acompaña a estos fetos y no a insuficiencia placentaria.

Pese a ello, no existe evidencia en la literatura que avale una conducta expectante en el manejo obstétrico de pacientes con trisomía 21 y flujo ausente o reverso en el estudio Doppler de la arteria umbilical fetal. Es más, esta reportado que fetos con aneuploidías y los hallazgos descritos al Doppler umbilical, tienen malos resultados perinatales, secundario a las malformaciones congénitas mayores que presentan estos fetos.

Expertos en medicina perinatal confirman los hallazgos en la literatura y manifiestan que éstas pacientes no deben ser manejadas en forma expectante.

\section{CONCLUSIÓN}

Concluimos que, si bien es posible encontrar fetos con trisomía 21 y Doppler umbilical con FDA/ FDR sin otra alteración en las pruebas de evaluación de bienestar fetal, éstos pacientes deben ser manejados en forma activa, como si se sospechara una insuficiencia placentaria. Recomendamos una evaluación exhaustiva de la anatomía cardíaca en fetos con aneuploidía que presenten FDA/FDR en la velocimetría Doppler de arteria umbilical, ya que una cardiopatía congénita pudiera ser la responsable de dicha alteración en los flujos sanguíneos fetales al Doppler. 


\section{BIBLIOGRAFÍA}

1. Vergani P, Andreotti C, Roncaglia N, Zani G, Pozzi E, Pezzullo JC, et al. Doppler predictor of adverse neonatal outcome in growth restricted fetus at 34 weeks' gestation or beyond. Am J Obstet Gynecol 2003;189:1007-11.

2. Alfirevic Z, Neilson JP. Doppler ultrasonography in high-risk pregnancies: systematic review with metaanalysis. Am J Obstet Gynecol 1995;172:1379-87.

3. Mégarbané A, Ravel A, Mircher C, Sturtz F, Grattau $\mathrm{Y}$, Rethoré $\mathrm{M}$, et al. The 50th anniversary of the discovery of trisomy 21: the past, present and future of research and treatment of Down syndrome. Genetics in Medicine 2009;11(9):611-6.

4. Sommer CA, Henrique-Silva F. Trisomy 21 and Down syndrome - a short review. Braz J Biol 2008;68(2):44752.

5. Wiseman F, Alford K, Tybulewicz V, Fisher E. Down syndrome - recent progress and future prospects. Human Molecular Genetics 2009;18(1):R75-R83.

6. Snijders RJ, Sherrod C, Gosden CM, Nicolaides KH. Fetal growth retardation: associated malformations and chromosomal abnormalities. Am J Obstet Gynecol 1993;168: 547-55.

7. Kurjak A, Kirkinen P. Ultrasonic growth pattern of fetuses with chromosomal aberrations. Acta Obstet Gynecol Scand 1982;61:223-5.

8. Krebs C, Macara LM, Leiser R, Bowman AW, Greer IA, Kingdom JC. Intrauterine growth restriction with absent end-diastolic flow velocity in the umbilical artery is associated with maldevelopment of the placental terminal villous tree. Am J Obstet Gynecol 1996;175:1534-42.

9. Salafia CM, Pezzullo JC, Minior VK, Divon MY. Placental pathology of absent and reverse end-diastolic flow in growth-restricted fetuses. Obstet Gynecol 1997; $90: 830-6$.

10. Rizzo G, Pietropoli A, Capponi A, Arduini D, Romanini C. Chromosomal abnormalities in fetuses with absent end-diastolic velocity in umbilical artery: analysis of risk factors for an abnormal karyotype. Am J Obstet Gynecol 1994;171:827-31.

11. Wong G, Levine D. Fetuses with trisomy 21 having conflicting findings on antenatal testing for fetal wellbeing. J Ultrasound Med 2005;24:1541-5.

12. Al-Gazali W, Chapman MG, Chita SK, Crawford DC, Allan LD. Doppler assessment of umbilical artery blood flow for the prediction of outcome in fetal cardiac abnormality. Br J Obstet Gynaecol 1987;94:742-5.

13. Goldhaber SZ, Brown WD, Sutton MG. High frequency of mitral valve prolapsed and aortic regurgitation among asymptomatic adults with Down's syndrome. JAMA 1987;258:1793-5.

14. Hsieh FJ, Chang FM, Ko TM, Chen HY, Chen YP. Umbilical artery flow velocity waveforms in fetuses dying with congenital anomalies. Br J Obstet Gynaecol 1988;95:478-82.

15. Meise C, Germer U, Gembruch U. Arterial Doppler ultrasound in 115 second and third-trimester fetuses with congenital heart disease. Ultrasound Obstet Gynecol 2001;17:398-402.

16. Dr. Cristian Belmar Jones, Especialista en Obstetricia y Ginecología, Profesor Auxiliar Escuela Medicina, Pontificia Universidad Católica de Chile, Estudios postdoctorales en Londres en las áreas de Cardiopatías Congénitas y Ecocardiografía Fetal.

17. Dr. Luis Medina Herrera, Especialista en Obstetricia y Ginecología, Unidad de Alto Riesgo Obstétrico, Complejo Asistencial Dr. Sótero del Rio, Staff en Centro de Diagnóstico e Investigaciones Perinatales (CEDIP), Estudios postdoctorales en Ecocardiografía anatómica y funcional en Perinatal Research Branch, National Institutes of Health (NIH).

18. Dr. Hernán Muñoz Salazar, Especialista en Obstetricia y Ginecología, Profesor Asociado Universidad de Chile, Vicepresidente de la Sociedad Chilena de Obstetricia y Ginecología, Miembro del Task Force of Fetal Echocardiography, International Society of Ultrasound in Obstetrics and Gynecology (ISUOG). 